\title{
Age-Related Hearing Loss and the Development of Cognitive Impairment and Late-Life Depression: A Scoping Overview
}

\author{
Rahul K. Sharma, B.S., ${ }^{1,2}$ Alexander Chern, M.D., ${ }^{1}$ and \\ Justin S. Golub, M.D., M.S. ${ }^{1}$
}

\section{ABSTRACT}

Age-related hearing loss (ARHL) has been connected to both cognitive decline and late-life depression. Several mechanisms have been offered to explain both individual links. Causal and common mechanisms have been theorized for the relationship between ARHL and impaired cognition, including dementia. The causal mechanisms include increased cognitive load, social isolation, and structural brain changes. Common mechanisms include neurovascular disease as well as other known or asyet undiscovered neuropathologic processes. Behavioral mechanisms have been used to explain the potentially causal association of ARHL with depression. Behavioral mechanisms include social isolation, loneliness, as well as decreased mobility and impairments of activities of daily living, all of which can increase the risk of depression. The mechanisms underlying the associations between hearing loss and impaired cognition, as well as hearing loss and depression, are likely not mutually exclusive. ARHL may contribute to both impaired cognition and depression through overlapping mechanisms. Furthermore, ARHL may contribute to impaired cognition which may, in turn, contribute to depression. Because ARHL is highly prevalent and greatly undertreated, targeting this condition is an appealing and potentially influential strategy to reduce the risk of developing two potentially devastating diseases of later life. However, further studies are necessary to elucidate the mechanistic relationship between ARHL, depression, and impaired cognition.

KEYWORDS: presbycusis, age-related hearing loss, depression, dementia, cognitive decline

${ }^{1}$ Department of Otolaryngology-Head and Neck Surgery, Columbia University Irving Medical Center, New York, New York; ${ }^{2}$ Columbia University Vagelos College of Physicians and Surgeons, New York, New York.

Address for correspondence: Justin S. Golub, MD, MS, Department of Otolaryngology-Head and Neck Surgery, Columbia University Irving Medical Center, 180 Fort Washington Ave, HP8, New York, NY 10032 (e-mail: justin.golub@columbia.edu).
Public Health Perspectives on Hearing Loss and Aging Outcomes; Guest Editor, Nicholas S. Reed, Au.D.

Semin Hear 2021;42:10-25. (C) 2021. Thieme. All rights reserved. Thieme Medical Publishers, Inc., 333 Seventh Avenue, 18th Floor, New York, NY 10001, USA DOI: https://doi.org/10.1055/s-0041-1725997.

ISSN 0734-0451. 
Presbycusis, or age-related hearing loss (ARHL), is a highly prevalent and undertreated condition, affecting more than two-thirds of adults older than 70 years. ${ }^{1}$ In recent years, researchers have identified an independent association between ARHL and both cognitive impairment and incident dementia. ARHL also is correlated with an increased risk of late-life depression; however, this relationship is less studied. $^{2-8}$

Both depression and cognitive impairment pose a major burden to worldwide healthcare systems. Dementia affects approximately 46.8 million people worldwide. Studies project that the prevalence will increase to 74.7 million by 2030 and to 131.5 million by 2050. ${ }^{9}$ The worldwide costs of dementia were estimated to be about $\$ 818$ billion in 2015 , and was projected to surpass $\$ 1$ trillion by 2018. ${ }^{10}$ Depression has a 12 -month and lifetime prevalence of $10.4 \%$ and $20.6 \%$, respectively, and affects more than 2 million of the 34 million Americans older than 65 years. ${ }^{11}$ Studies have projected that this condition will be the major cause of increased health care costs in middle-to-higher income countries by the year 2030. ${ }^{12}$

The relationship between ARHL and conditions of aging raises the exciting possibility that hearing loss, a common and undertreated condition, could be a modifiable risk factor. Though no causal mechanisms have been definitively established, plausible mechanisms link ARHL to both depression and cognitive impairment. Targeting ARHL could potentially reduce the prevalence, burden, or healthcare costs for these difficult-to-manage diseases of late life. ${ }^{13}$ Treatment is available for all levels of ARHL, ranging from hearing aids for mild-tomoderate hearing loss to cochlear implants (CIs) for severe-to-profound hearing loss. ${ }^{14}$ Certain rare forms of hearing loss can be improved with minor surgery. However, despite the overwhelming high prevalence of ARHL in the population, available treatments are grossly underutilized. This review summarizes the associations between ARHL, cognition, and depression, and highlights the importance and potential benefits of further investigating these relationships.

\section{BACKGROUND OF AGE-RELATED HEARING LOSS}

ARHL is inherent in human aging: given a long enough life, nearly everyone will develop hearing loss. ARHL is characterized as a gradual, progressive, and bilateral symmetric sensorineural hearing loss that initially affects higher frequencies (pitches). It is the second most common condition in the geriatric population. ARHL is a multifactorial condition that manifests due to a combination of intrinsic factors, such as genetic predisposition, and extrinsic factors, such as environmental noise exposures, cardiovascular disease, and ototoxic medications. The pathophysiology is attributed to degenerative changes of inner ear, including loss of cochlear inner and outer hair cells, degradation of spiral ganglion cells, or atrophy of the stria vascularis. ${ }^{15}$ However, the underlying trigger of this degenerative process remains unknown. Unfortunately, ARHL is irreversible and treatments solely aim to maximize remaining function (hearing aids) or completely bypass the inner ear and directly stimulate the hearing nerve (CIs).

ARHL initially presents as increased hearing thresholds, meaning that sounds are heard at a decreased volume. The quietest level (in decibel hearing level, or $\mathrm{dB}$ ) at which the subject can hear the sound is termed the hearing threshold. Hearing is tested and hearing thresholds are identified through pure tone audiometry, where tones of a specific frequency are presented in a soundproof booth. The tones progressively increase in intensity (i.e., loudness) and the individual notes when he/she can hear the sound. Once pure tone hearing thresholds are obtained, type, degree, and configuration of hearing loss can be determined.

ARHL initially affects higher frequencies but progresses to involve midrange and lower frequencies over time. Speech primarily falls within the midrange of frequencies. Certain parts of words, such as the consonant $/ \mathrm{t} /$ in the word "right," fall among high frequencies. With ARHL, consonants are mostly affected, and can be misheard or not heard at all. While this may seem like a clarity problem, this issue stems from lack of volume. Hearing aids are an effective treatment, as they can be manipulated 
to preferentially increase the loudness of certain frequencies.

Over time, the clarity of sound also will decrease. This can be tested with speech audiometry, where a individual is asked to repeat words at a comfortable level of loudness. The word recognition score (sometimes called the "speech discrimination score") is the percent of correctly repeated words. When clarity and word recognition are affected, individuals with ARHL particularly struggle with conversations in background noise and music enjoyment. The challenge of understanding speech in background noise is significant, as most speech in the real world occurs in the presence of some type of competing sound. These difficulties may decrease participation in social activities $^{16}$ and reduce quality of life. ${ }^{17}$

Hearing loss is a major burden for the elderly population. Over two-thirds of patients older than 70 years are afflicted, and the prevalence will only increase as the population ages. ${ }^{1}$ Evidence shows that hearing interventions (i.e., hearing aids and CIs) improve both hearing-specific quality of life as well as overall quality of life. However, despite the high prevalence of hearing loss in the U.S. population, fewer than $20 \%$ of adults with hearing loss use any treatment. ${ }^{14}$

\section{AGE-RELATED HEARING LOSS AND COGNITION}

Dementia, the most concerning form of cognitive impairment, is a debilitating condition with extraordinary societal costs and no known treatment. There is great effort to identify and target modifiable risk factors of dementia, which could reduce or delay the burden of the disease later in life. These include smoking, cardiovascular disease, diabetes, and obesity. Hearing loss is a relatively new recognized modifiable risk factor. ${ }^{18}$ Multiple cross-sectional studies have identified associations between ARHL and both cognitive impairment and dementia, controlling for several potentially confounding risk factors. ${ }^{19}$ Similarly, longitudinal studies have identified an independent association between hearing loss and incident dementia, also after controlling for potential confounders. One noteworthy study found that the risk of dementia over a 12-year period was 1.9 times higher among those with mild hearing loss compared with those with normal hearing. The risk among those with severe hearing loss increased by nearly five times. ${ }^{3}$

The potential impact of treating hearing loss is substantial, as was illustrated by using population-attributable fractions (PAFs). This metric can be used to estimate the percent reduction in incident dementia over a given time if a specific risk factor was removed. The unweighted PAF of hearing loss for dementia was recently calculated to be $23 \%$. This is higher than all other PAFs of other modifiable risk factors, such as low education, depression, social isolation, hypertension, diabetes, and smoking. ${ }^{18}$ Considering the prevalence of hearing loss in the elderly, as well as the availability and accessibility of treatment options, targeting hearing has recently become an attractive strategy for potentially reducing the risk or delaying the onset of dementia.

In addition to dementia, several cohort studies and meta-analyses have noted a relationship between hearing loss and cognitive impairment. Specifically, ARHL measured through pure tone audiometry has been associated with worse scores on a wide range of neuropsychological tests assessing several cognitive domains, including memory, language, and executive function. ${ }^{20}$ This has been consistently observed in both small and large observational studies ${ }^{21-23}$ as well as cross-sectional $^{24}$ and longitudinal populationbased studies. ${ }^{25}$ One newer study showed that there was no hearing threshold above which the hearing-cognition relationship begins. Subclinical hearing loss, defined as imperfect hearing $(>0$ $\mathrm{dB})$ that is better than the $25-\mathrm{dB}$ threshold for mild hearing loss, was still a risk factor for impaired cognition. ${ }^{26}$

Several preliminary noncontrolled treatment trials have suggested an improvement in cognition with hearing loss treatment. Studies reported better scores in a wide range of neurocognitive functions associated with both memory and executive function. ${ }^{27,28}$ While these studies have several flaws, including potential confounding, their results are encouraging and provide evidence for a positive impact of hearing loss treatments on cognitive function. 
The association between ARHL and both cognitive impairment and dementia has been increasingly substantiated by a growing literature; however, the mechanism behind this connection is uncertain. Several plausible mechanisms elucidating the hearing loss-cognition relationship have been suggested. These mechanisms can be broadly divided into causal (ARHL causes cognitive impairment) or common (factors that cause both ARHL and cognitive impairment) mechanisms. ${ }^{29,30}$

\section{Casual Mechanisms}

Causal mechanisms indicate that ARHL, through various potential mediating pathways, increases the risk of cognitive impairment or dementia. Several of these potential pathways have been proposed (Fig. 1, pathways A-C).

\section{COGNITIVE LOAD}

Hearing loss can lead to a greater cognitive load (Fig. 1, Pathway A), as cognitive resources are expended from processing a degraded auditory signal. Consequently, cognitive reserve can be depleted. ${ }^{31}$ Individuals with better cognitive reserve have an easier ability activating and reallocating compensatory processes when suffering from abnormal brain function. This phenomenon is thought to occur in patients with brain damage from trauma or stroke, as those with less cognitive reserve are less effectively able to maintain function of the damaged region compared with those with more cognitive reserve. $^{32}$
This idea may extend to other pathologies that increase the brain's cognitive load, such as hearing loss. Those who experience hearing loss may consume cognitive resources from other brain regions while struggling to understand speech. This could lead to cognitive dysfunction if cognitive reserve is not abundant in the affected individual. Several meta-analyses and systematic reviews have shown that ARHL impacts a wide range of cognitive domains, including executive function, word understanding, attention, processing latency, short- and long-term memory, and working memory. ${ }^{33}$ Brain activity changes on functional magnetic resonance imaging (fMRI) in postlingually hearing-impaired adults. Increased activation of frontal and prefrontal cortical regions coupled with decreased activation in the temporal region (commonly associated with auditory processing) on speech-stimulated EEG supports the idea of increased recruitment of other brain regions to aid speech perception. In these studies, the process of resource allocation in individuals with hearing loss likely results in increased cognitive load, as those with hearing loss are redirecting finite cognitive resources to decode speech, which previously (i.e., prior to hearing loss) was not a major consumer of the brain's cognitive reserve. ${ }^{19}$ As a result, there are not enough cognitive resources left to devote toward other higher-level processes such as memory and executive function. This could contribute to cognitive decline.

Anecdotally, direct experiences of hearingimpaired older adults support this theory. In challenging listening environments, understanding

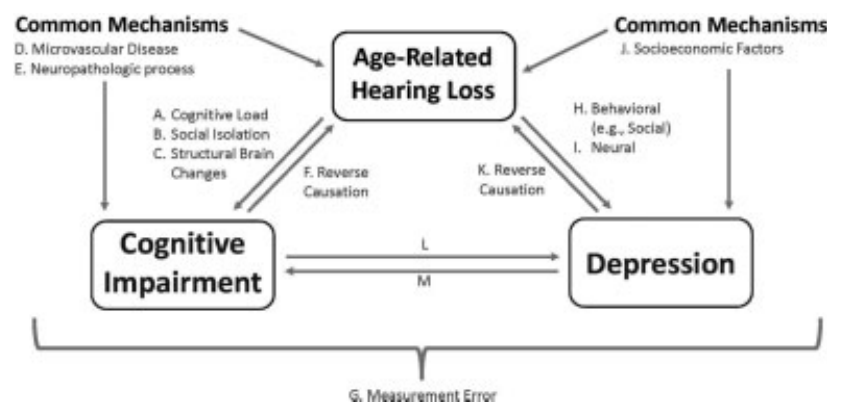

Figure 1 Proposed mechanistic relationship between age-related hearing loss, cognitive impairment, and depression. Common mechanisms are also called confounders. Factors that appear along an arrow between hearing loss and cognitive impairment (cognitive load, social isolation, brain changes) or between hearing loss and depression (behavioral, neural) are also called mediators. 
words a speaker is saying can require greater cognitive effort. For example, the speaker may have said "cat" or "rat" but based on the subsequent sentence, neither makes sense. "Bat" becomes the likelyword in this scenario. Though by the time, the listener has processed this, the speaker is three sentences ahead and the entire purpose of the story is lost. ${ }^{34}$

\section{SOCIAL ISOLATION}

Social isolation (Fig. 1, Pathway B) is another possible mediator that could explain a causal relationship between ARHL and cognitive impairment. ${ }^{35,36}$ Social connectedness includes many different factors, including living arrangements, depth and size of social networks, participation in social gatherings, and engagement in community activities. ${ }^{37}$

Hearing loss may adversely affect socialization. Those suffering from hearing loss require more cognitive energy for verbal conversation $^{38,39}$ and thus communication may suffer. Relationships can be negatively affected and activities that were once enjoyed, such as interacting in groups or attending social events, may no longer be pleasant. Those affected by hearing loss may feel that they are burdening others, as conversations require more patience from those who are not. Additionally, hearing loss has been shown to reduce an individual's ability to feel self-confident and independent, which can hamper interactions with less familiar, outside communities. The combination of these various factors can result in a feeling of loneliness, disconnection from previously strong social circles, and a reduction in social engagement.

Poor social engagement is independently associated with a higher risk of cognitive decline. ${ }^{40}$ Promoting social engagement allows the older individual to better cope with the aging process. Diminished social networks and engagement increase the rate of cognitive decline through reduced brain stimulation. Additionally, people who feel disconnected from their community are less likely to participate in activities such as physical exercise and routine healthcare visits. ${ }^{41}$ This might also adversely affect cognitive function. Treating hearing loss, primarily through hearing aids, has already been shown in both cross-sectional and longitudinal studies to improve feelings of loneliness and quality of life. $^{42,43}$

\section{STRUCTURAL BRAIN CHANGES}

ARHL may lead to changes in brain structure (Fig. 1, Pathway C) which in turn may increase the risk of cognitive impairment and dementia. This association has primarily been observed through the measurement of brain volumes on MRI. ${ }^{29}$ Cross-sectional studies have noted decreased volume of the primary auditory cortex (i.e., in the temporal lobe) on MRI in patients with ARHL. This is likely a result of reduced activation of the region due to the lack of stimulation from the afferent auditory system. ${ }^{44,45}$ Longitudinal studies also have found that individuals with hearing loss had an increased rate of brain volume reduction compared with those who did not suffer from hearing loss. ${ }^{46}$ Notably, the rate of brain volume decline in patients with ARHL was elevated for the entire cerebral cortex and the temporal lobe, which is responsible for speech and language processing. Atrophy of the temporal lobe is also seen in early stages of Alzheimer's disease, as this region is responsible for different forms of memory and sensory transmission between different structures. ${ }^{47}$ It is possible that ARHL accelerates cognitive decline by contributing in an additive manner to the atrophy of the temporal lobe and other regions commonly associated with Alzheimer's. Prospective cohort studies have identified a relationship between midlife hearing impairment and reduced brain volume trajectories of the temporal lobe. ${ }^{48}$

\section{Common Mechanisms}

Another possibility is that ARHL is related to cognitive impairment but does not cause it. Instead, confounding processes may contribute to the development of both ARHL and cognitive impairment. Two possible mechanisms are microvascular disease and neuropathologic processes.

Microvascular disease (Fig. 1, Pathway D) has been implicated as a cause of both ARHL and dementia. Chronic damage to the microcirculation of the cochlea can result in hearing impairment. ${ }^{49}$ Disease of small vessels results in diminished cochlear blood flow, which 
subsequently leads to ischemic intracochlear injury. The stria vascularis, which is responsible for regulation of endolymph (an inner ear fluid) is fundamental for proper cochlear physiology and is the most notable location of ischemic injury. ${ }^{50}$ Clinical studies have found associations between stroke and acute sensorineural hearing loss, as well as chronic ARHL, likely because microvascular disease causes both. ${ }^{51}$

Microvascular cochlear damage is analogous to vascular dementia, in which periodic ischemic damage to the major regions of the brain due to diseased small vessels leads to cognitive decline. This is secondary to both local damage of major brain regions supplied by damaged vasculature and induction of neurodegenerative changes in distant regions. This inevitably leads to derangements in structural connectivity which results in impaired communication between cortical and subcortical brain regions. ${ }^{52-54}$

Recent studies that have shown an association between ARHL and impaired cognition have adjusted for vascular risk, because of the obvious confounding potential of vascular disease. Conditions such as hypertension, smoking, and diabetes have been controlled for in multivariable regression. Since the relationship between ARHL and impaired cognition persists even after accounting for this known common cause, then vascular disease cannot entirely account for the relationship. ${ }^{5,7}$

Basic neuropathologic processes could, hypothetically, cause both dementia and ARHL (Fig. 1, Pathway E). Classic histopathologic features underlying Alzheimer's include senile plaques (i.e., $\beta$-amyloid deposits) and neurofibrillary tangles (i.e., hyperphosphorylated tau protein aggregates) on autopsy specimens of the brain. The presence of these features on pathology specimens is tightly connected to dementia. ${ }^{55}$ In theory, amyloid and tau buildup also could cause ARHL by depositing in key auditory structures, such as the central auditory cortex, cochlear nuclei, inferior colliculi, and thalamus. ${ }^{56}$ However, ARHL is a peripheral phenomenon diagnosed by pure tone audiometry and such deposits have yet to be identified in the peripheral auditory system. Neuroimaging studies have shown that poorer hearing is associated with poorer cognitive function, but not with amyloid deposition. ${ }^{57}$ Though further studies are needed to confirm, this may suggest that the mechanism is independent of amyloidrelated brain changes.

A possibility remains that undiscovered factors cause both ARHL and dementia (Fig. 1, Pathway E). Because they are undiscovered, they cannot be controlled for in observational studies. For example, it is theoretically possible that some unknown neurodegenerative process causes both ARHL and dementia, a phenomenon known as residual confounding. A randomized controlled trial would be needed to eliminate the possibility of residual confounding.

\section{Reverse Causation}

Scenarios where impaired cognition or dementia cause accelerated hearing loss (Fig. 1, Pathway F) are theoretically possible, but unlikely. This could occur if cognitively impaired individuals tend to be exposed more frequently to excessively loud noise or ototoxic substances than individuals without cognitive impairment. A lower level of health literacy is associated with cognitive decline, ${ }^{58}$ meaning those with cognitive impairment may not as effectively understand how to preserve hearing through avoidance of perpetual noisy environments or avoidance of medications. However, longitudinal studies have shown that hearing loss increases the risk of later cognitive impairment and dementia. ${ }^{3}$ This temporal association is incompatible with reverse causation.

\section{Measurement Error}

There is also the concern that hearing loss is associated with inaccurate diagnosis of poor cognition. Poor performance on the cognitive test may be due to instructions not being heard (Fig. 1, Pathway G). Many cognitive tests, including the commonly used screening Mini Mental Status Exam (MMSE) and Montreal Cognitive Assessment (MoCA), rely on the patient's ability to hear, as instructions are usually verbally communicated. An inability to hear the instructions may lead to an artificially low score on cognitive testing, and therefore the extent of cognitive impairment may be 
overestimated. This is unlikely, as subjects are usually tested by a trained examiner in a quiet room where instructions, unless the individual has severe hearing loss, are likely audible. The association between hearing loss and poor cognition persists even when those with severe hearing loss are eliminated. One recent study found an association between cognitive impairment and subclinical hearing loss. ${ }^{26}$ Moreover, studies have been conducted using nonverbal cognitive tests and have found an association between ARHL and reduced cognitive functioning. ${ }^{59}$

Cognitive impairment could make it difficult to appropriately and reliably perform a hearing test. This could result in an artificially low hearing test score that is unrelated to actual hearing ability. This is unlikely due to the simplicity of the clinical audiometric battery, which consists of raising a hand when a tone is heard or repeating words. Examiners are trained to adjust the speed of the test, which allows subjects to have ample time to process each step. Pure tone audiometry, the primary hearing measure in most current epidemiologic studies, is designed to test peripheral hearing. Higher cortical processes that would normally be diminished in individuals with dementia, however, are not assessed by pure tone audiometry. ${ }^{60}$ As a proof of point, pure tone audiometry can be completed in children as young as 3 years old. Patients who experience severe dementia might struggle with audiometry testing. For this reason, previous longitudinal studies have excluded those with severe dementia at baseline. ${ }^{61}$

\section{Other Limitations}

While studies have shown enough evidence to strongly hypothesize a causal relationship between ARHL and dementia, it is likely that the relationship is multifactorial, involving several different mechanisms that could act in an additive manner. The ability to make concrete conclusions is limited by the difficulties found in studying cognition and hearing loss. For example, in studies using exposure and outcome measures which are not necessarily ideal, it is possible that there is overdiagnosis of dementia in those with hearing loss, or vice versa. Hearing loss may give the illusion of cognitive decline in those affected. As a result, family members or health care providers may more aggressively screen for dementia and therefore identify the disease earlier and more frequently than those not affected with hearing loss. However, this phenomenon has not been formally explored.

\section{Additional Considerations}

In addition to hearing loss, other sensory impairments have been associated with cognitive decline, including both visual and olfactory impairment. ${ }^{62,63}$ The visual-cognition mechanism may resemble the hearing-cognition mechanism. Visual impairment could lead to social isolation, as well as reallocation of cognitive resources, due to a lack of stimulation of the primary visual cortex.

The olfaction-cognition relationship, particularly olfaction-dementia, may operate in the reverse direction, such that dementia is the cause as opposed to the effect. This theory is primarily supported by the presence of neurofibrillary tangles in the olfactory bulb in both Alzheimer's and degenerative diseases of the brain. ${ }^{64}$ This is in contrast to the peripheral auditory mechanisms, where such pathology has not been seen. Further studies exploring the effect of confounding sensory impairment, as well as causation, will need to be conducted before concrete conclusions can be made.

Vestibular function and balance also have been associated with cognitive function. Crosssectional studies and longitudinal studies have illustrated that poorer balance is associated with worse performance on cognitive testing after controlling for confounders, including hearing loss. ${ }^{65,66}$ The exact nature of this relationship requires further study.

The relationship between the other senses, such as taste and touch, and cognitive decline remain largely unstudied.

\section{AGE-RELATED HEARING LOSS AND DEPRESSION}

Elderly individuals experiencing hearing loss have higher levels of depression and score worse on geriatric depression scales compared with their normal hearing counterparts. ${ }^{8,67}$ There are multiple theories that may explain why hearing 
loss is associated with late-life depression. Individuals who have difficulty hearing also have difficulty following conversations. This can be frustrating when interacting with family and friends, and can diminish their enjoyment of social activities and relationships.

Hearing loss also makes it difficult for those affected to participate in leisure activities, primarily because they feel less comfortable physically navigating through their environment without hearing. This could lead to the development of depressive symptoms. Studies suggest that those with significant hearing loss report lower and impaired physical activity as well as slower gaits. ${ }^{68}$ Symptoms such as decreased leisure activity, slowed gait, and poor physical performance are related to frailty, a condition of generalized physiologic decline associated with increased morbidity and mortality risk exhibited by elderly adults. Interestingly, such symptoms of the frailty phenotype also are exhibited by individuals with late-life depression. Researchers have proposed that there is a bidirectional association with characteristics of frailty and late-life depression. ${ }^{69}$

There are not as many high-quality studies that concretely establish the relationship between hearing loss and depression as there are in the cognitive literature. Studies examining the relationship between depressive symptoms and hearing loss have demonstrated mixed results. This may be attributed to the variability in measurement strategies as well as the weakness in study designs. One study identified a relationship between mild hearing loss and depression, but not moderate or severe hearing loss. ${ }^{70}$ Interestingly, another study identified a linear relationship between the degree of depression and greater hearing deficit. ${ }^{71}$ Yet another found an association between pure-tone audiometry hearing thresholds and depression, but not with self-reported hearing loss. ${ }^{72}$ There have been consistent findings of an association between hearing aid use and a reduced odds of depression or depressive symptoms. ${ }^{73,74}$ This phenomenon also has been described in individuals with cochlear implants. ${ }^{75}$

While results have varied, there are several potential mechanisms by which hearing loss may contribute to the development of depression. These can be divided into behavioral factors and neural pathways.

\section{Causal Mechanisms}

\section{BEHAVIORAL MECHANISMS}

Behavioral mechanisms elucidating an association between hearing loss and depression are relatively intuitive (Fig. 1, Pathway $\mathrm{H}$ ). Hearing is fundamental to communication via spoken language; thus, hearing loss is inextricably related to social dysfunction. Individuals with hearing impairment are more likely to withdraw from society, experience social isolation, and have reductions in social networks. These factors, in turn, have been independently associated with depression. ${ }^{76-78}$ This social isolation primarily stems from an impairment in communication. Without effective communication, individuals are less able to maintain relationships, social activities, and leisure activities. ${ }^{79}$

Social isolation is highly correlated with depression, in addition to poor quality of life and increased mortality and morbidity. ${ }^{80-82}$ As outlined earlier, reduced social interaction is associated with an increased risk of incident dementia, which has been linked, in turn, to the development of depressive symptoms, independent of other confounders such as hearing loss and other sensory impairment. ${ }^{40,83}$ Self-reported loneliness, a common component of the social isolation, also has been a reported predictor of the development of Alzheimer's disease in longitudinal studies. ${ }^{76}$

Hearing loss also has been associated with poor physical functioning and activity. This includes slower gaits and substandard execution on measures of physical performance. ${ }^{68,84,85}$ Additionally, studies have identified an independent inverse relationship between hearing and postural control, which can be associated with difficultly walking and a higher risk of falls. ${ }^{86,87}$ A relationship between falls and hearing loss has been well-documented. In fact, a systematic review found that the odds of falling were 2.39 times greater for elderly patients with hearing loss. ${ }^{88}$ This review, however, admits the statistic is limited by potential publication bias. Furthermore, the important confounding effect of poor vestibular function (i.e., since hearing 
and vestibular function are often related) was generally not controlled for. This is an important consideration, as hearing aids rehabilitate the cochlear portion of the inner ear, not the vestibular portion. The fear of falling has been associated with social isolation, anxiety, a reduced level of independence, and depression. ${ }^{89}$ Finally, decreased physical activity and impaired gait are part of the syndrome of frailty. Researchers have proposed that there is a bidirectional relationship between frailty syndrome and late-life depression.

Auditory impairment is closely associated with tinnitus, an unwanted and often bothersome sound most commonly described as ringing. ${ }^{90}$ While the etiology of tinnitus is complex, hearing loss is partly causative. Indeed, hearing loss is diagnosed in more than $90 \%$ of patients with tinnitus. ${ }^{91}$ The reported prevalence of bothersome tinnitus in individuals older than 60 years ranges from 8 to $20 \%{ }^{92}$ Chronic tinnitus is associated with depression and anxiety symptoms, and is likely a component of the mechanisms underlying hearing loss and depression. Bidirectional, or circular, association is probable as tinnitus can cause worsening anxiety or depression, which increases the annoyance of tinnitus. Chronic tinnitus has been associated with suicide attempts, although this relationship is still under investigation. ${ }^{93}$

\section{NEURAL MECHANISMS}

Neuroimaging studies support a relationship between ARHL and depression (Fig. 1, Pathway I). As described previously, hearing loss is associated with several brain changes in both the peripheral and central auditory pathways, as well as other related structures of the prefrontal cortex over time. ${ }^{94}$ In studies using MRI imaging, the brains of patients with ARHL have demonstrated diminished volumes of many brain regions, including the temporal and frontal cortices, amygdala, hypothalamus, and primary auditory cortex. ${ }^{44,45,95,96}$ Many mental health symptoms, including those seen in depression, have been linked to the neuronal connectivity of these brain regions. ${ }^{97}$ Therefore, pathophysiological changes of neurological pathways and atrophy of key structures due to ARHL may contribute to the development of depression.
In addition to brain volume changes, fMRI studies have shown functional changes in the brains of depressed patients. Based on these functional changes, some believe it is possible to subclassify depression based on activity differentials. ${ }^{98}$ Reductions in activity of the thalamus, temporal lobe, cerebellar posterior lobe, insula, and occipital lobe have been observed in depressed patients relative to healthy patients. ${ }^{99}$ Studies have shown that these functional abnormalities persist even after treatment. ${ }^{100}$ Therefore, hearing loss may contribute to the development of late-life depression through compensatory recruitment of other areas of the brain, such as those necessary for emotional stability, to support effortful listening. These can lead to overtaxing the cognitive network's capacity and cause executive dysfunction, which is a classic characteristic of depression in the elderly. Diminished executive function predicts a poorer response to antidepressant medications, and is associated with a higher rate of relapse and recurrence of major depression. ${ }^{101}$ Studies have suggested that individuals with greater cognitive reserve may be better equipped to devote more energy toward brain regions that are responsible for regulating emotion. ${ }^{102}$ Therefore, as described earlier, depletion of cognitive reserve due to hearing loss can contribute to the development of depression in a similar manner to dementia. ${ }^{32}$ Notably, studies have shown deactivations in limbic structures in those with hearing loss. These structures are highly relevant for the experience of emotionality, and normally have strong neural connections with the auditory processing centers of the brain. ${ }^{103}$ Dysregulation of these limbic structures in ARHL patients may result in downstream effects (i.e., emotional to the idea that hearing loss could have a direct mechanism with depression).

\section{Common Causes}

Certain factors may contribute to the development of both depression and ARHL independently. For example, both lower socioeconomic status (Fig. 1, Pathway J) and low education level are risk factors for the development of depression. ${ }^{104}$ These factors also could contribute to ARHL, in theory, if these patients are 
more likely to be exposed to noisier environments and more ototoxic medications. However, high-quality studies have attempted to control for such sociodemographic factors with multivariable regression and the hearing-depression relationship has persisted. Yet, residual confounding, whereby confounders that we are not aware of or cannot control for, is still possible. To fully control for confounders, a randomized control trial is needed.

\section{Reverse Causation}

Depression could contribute to poorer hearing in a reverse mechanism. This would be the case if those who were depressed were more likely to expose themselves to environments with excessive noise, or expose themselves to more ototoxins than those who are not depressed. This is unlikely through the noise-induced pathway, as studies looking at hearing loss in individuals with depression do not observe the classic hearing loss at a frequency of $4 \mathrm{kHz}$ seen in those with noise-induced hearing loss. ${ }^{105}$ Furthermore, ARHL is only partly due to environmental factors (i.e., preventable), as opposed to intrinsic or genetic hearing loss.

\section{Measurement Error}

Substandard performance on depression testing may not reflect depression in an individual, but rather could be a result of inability to hear the testing instructions. In a similar fashion to tests of cognition, those with hearing loss may artificially do worse on depression testing due to the inability to hear questions or instructions. This is unlikely, as several of the common screening tests for depression, such as the PHQ-9, are written tests. Furthermore, the relationship between hearing and depressive symptoms persists even if individuals with severe hearing loss are excluded.

Finally, individuals with depression may do worse on a hearing test not because of worse hearing, but because they were more apathetic and did not expend enough effort on the test. Hearing tests are reliable and relatively easy to complete. As described earlier, patients as young as 3 years can complete hearing assess- ments, illustrating the degree of effort necessary to complete the test.

\section{Other Limitations}

Many of the studies listed earlier utilize selfreported data for both hearing and depression. This can introduce bias due to the stigma associated with functional impairment, preventing patients from consciously or subconsciously choosing to report their conditions. Additionally, studies vary greatly in how they choose to measure depression, as some do not use quantitative scales. Some studies utilize depressive symptom inventories; however, this measures depressive symptoms as opposed to clinically diagnosed depression. Few studies use psychometrically valid assessments.

Studying depression in the older adults can be difficult, as it can manifest in ways that may not be measured using standard depression inventories. For example, depression can commonly manifest as somatic symptoms in older patients. Lethargy, one of the most common symptoms of depression, may not be picked up by conventional screening methods. ${ }^{106}$ Another key consideration is the temporal variability of depressive symptoms. Unlike dementia, which is generally irreversible and progressive, depression can be episodic. Symptoms will fluctuate depending on treatment with antidepressants, interpersonal or social support, and unknown factors. This makes it difficult to sensitively capture depression, especially when using crosssectional designs. Due to the variability of depression presentation, it would be methodologically beneficial to limit studies to a relatively uniform population. For example, this could be done by studying ARHL in patients with clinically diagnosed depression who receive selective serotonin reuptake inhibitor treatment, as patients must have a certain intensity of symptoms to indicate medical treatment. ${ }^{101}$

Some have attempted to study how the treatment of hearing loss is related to depression. However, it is difficult to establish causality between hearing devices and depression, especially with cross-sectional studies, as those obtaining hearing aids or CIs are more motivated and less likely to be depressed. One study showed the opposite of an expected effect, in 
which hearing aid use was associated with an increased risk of social isolation. The author notes that the study was limited by a questionnaire for social isolation with a low sensitivity, which is not an uncommon problem in many studies. ${ }^{42}$ The authors speculate that hearing aids discourage engagement in social activities due to the amplification of background noise in busy locations, as well as the stigma associated with wearing the device. Fortunately, hearing aids are immediately reversible, and individuals can always remove them if they negatively affect hearing ability, or perceived cosmesis, in certain situations.

\section{THE NEXUS OF ARHL, DEPRESSION, AND COGNITION}

Hearing loss has been independently associated with both depressive symptoms and cognitive impairment. However, these relationships do not act in silos. Depression is a risk factor for cognitive impairment, while cognitive impairment is a risk factor for depression. Thus, a cycle could be feasible where depression exacerbates cognitive function which further exacerbates depression (Fig. 1, Pathways L-M).

As previously described, the hearing-cognition and hearing-depression relationships share mediators, including social isolation (Fig. 2). Treating hearing loss would likely have a beneficial effect on socialization because of this common mechanistic pathway. This treating hearing loss, in turn, could have a downstream effect on both cognition and depression.

Likewise, structural brain changes are also a common mediator. ${ }^{33}$ Brain volume changes have been seen in both depression and dementia. ${ }^{107,108}$ Reduction in volume of several major cortices such as the postcentral cortex, insula, parietal, occipital, hippocampus, amygdala, and orbitofrontal lobes have been seen in patients diagnosed with major depressive symptoms. ${ }^{109,110}$ Similarly, dementia is associated with several brain changes throughout the cerebral cortex, including regions of memory and emotional processing, such as the prefrontal, temporal, and parietal lobes; anterior cingulate gyrus; limbic structures; and insula. ${ }^{46,108}$ In theory, hearing loss could contribute to both dementia and depression via brain volume changes, as many structures commonly atrophied in both conditions are not mutually exclusive.

Finally, hearing loss could cause both depression and dementia through a common mediating mechanism of depletion of cognitive reserve; fMRI studies have shown decreased activity in multiple cortical regions in patients diagnosed with depression, dementia, and hearing loss. ${ }^{111}$ Similar to brain volume changes, the regions shown to change in activity in both dementia and depression are not mutually exclusive. By devoting more cognitive energy to hearing, cognitive reserve devoted to both cognition and emotional regulation could get depleted, which would result in both depressive symptoms and cognitive decline. However, it is still unclear whether depression and dementia are the causes or consequences of brain changes. ${ }^{112}$

If mechanisms underlying ARHL and dementia, as well as ARHL and depression, exist, then clinicians may be able to target both at once.

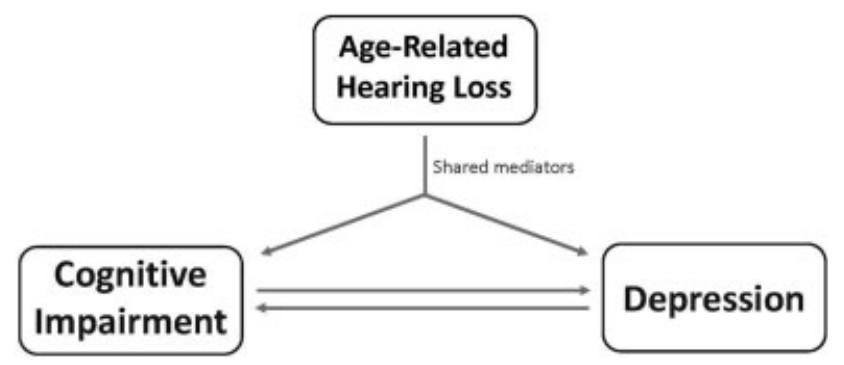

Figure 2 Additional mechanistic detail showing the interrelationship between age-related hearing loss, cognitive impairment, and depression. 


\section{FUTURE DIRECTIONS}

ARHL has been independently associated with both cognitive impairment and depression. While the mechanism between the associations is unclear, studies have identified hearing loss as a potential modifiable risk factor for these highly prevalent and burdensome conditions.

Considering the high prevalence and undertreatment of ARHL, further exploring the effect of hearing loss treatment on depression and cognition should be expedited. Studies have suggested that hearing loss treatment, including hearing aids and CIs, may be effective for the treatment of both dementia ${ }^{113,114}$ and depression. ${ }^{42,73,75}$ While promising results may be found, these studies must be approached with caution because of design limitations.

Current evidence suggests it is possible that aggressive utilization of hearing aids could have substantial health and cost-benefit advantages. These advantages could include the treatment of ARHL and quality of life as well as the symptoms of dementia and depression. ${ }^{115}$ Medicare does not currently cover hearing aids, but modeling suggests that utilization of hearing aids would reduce Medicare spending. ${ }^{116}$ With that said, hearing aids are accessible, and devices will be less expensive due to the recent passage of the US Over the Counter Hearing Aid Act of 2017. This new regulatory change will allow individuals with perceived mild-to-moderate hearing loss to purchase hearing aids over the counter in the United States, without a prescription. ${ }^{117}$

Further studies must be conducted before treatment guidelines can be firmly established, primarily through randomized clinical trials, as these are needed to infer causation. The evidence base today is largely from cross-sectional and longitudinal studies. The use of neuroimaging can be a strong tool for observing modifications in cortical activity and structure as aural rehabilitation technology is used.

As further evidence implicates hearing loss in impaired cognition and depression, strategies to expand access to hearing loss treatment and technology would be beneficial. Healthcare workers who are not otolaryngologists or audiologists largely ignore hearing loss. They must be educated about the relationships between ARHL, cognitive impairment, and depression.
By doing so, primary care physicians may begin to routinely recommend hearing testing and treatment for their older patients. Likewise, psychiatrists may consider targeting hearing loss as a manageable component of depression.

\section{CONCLUSION}

Age-related hearing loss is a potential risk factor for cognitive impairment, dementia, and late-life depression. It is a highly prevalent and undertreated condition that is a potential target and modifiable risk factor in preventative strategies for both dementia and depression. Additional research, particularly randomized control trials and large prospective studies incorporating more neuroimaging modalities, are needed to further elucidate possible causal mechanisms behind the associations observed, and to comprehensively inform treatment guidelines.

\section{CONFLICTS OF INTEREST}

J.S.G. received travel expenses for industrysponsored meetings (Cochlear, Advanced Bionics, Oticon Medical) and consulting fees or honoraria (Oticon Medical, Auditory Insight, Optinose, Abbott, Decibel Therapeutics), and also the department received unrestricted educational grants (Storz, Stryker, Acclarent, 3NT, Decibel Therapeutics).

R.K.S.: None.

A.C.: None.

\section{REFERENCES}

1. Lin FR, Thorpe R, Gordon-Salant S, Ferrucci L. Hearing loss prevalence and risk factors among older adults in the United States. J Gerontol A Biol Sci Med Sci 2011;66(05):582-590

2. Herbst KG, Humphrey C. Hearing impairment and mental state in the elderly living at home. BMJ 1980;281(6245):903-905

3. Lin FR, Metter EJ, O'Brien RJ, Resnick SM, Zonderman AB, Ferrucci L. Hearing loss and incident dementia. Arch Neurol 2011;68(02): 214-220

4. Gurgel RK, Ward PD, Schwartz S, Norton MC, Foster NL, Tschanz JT. Relationship of hearing loss and dementia: a prospective, populationbased study. Otol Neurotol 2014;35(05):775-781 
5. Golub JS, Luchsinger JA, Manly JJ, Stern Y, Mayeux R, Schupf N. Observed hearing loss and incident dementia in a multiethnic cohort. J Am Geriatr Soc 2017;65(08):1691-1697

6. Uhlmann RF, Larson EB, Rees TS, Koepsell TD, Duckert LG. Relationship of hearing impairment to dementia and cognitive dysfunction in older adults. JAMA 1989;261(13):1916-1919

7. Deal JA, Betz J, Yaffe Ket al;Health ABC Study Group. Hearing impairment and incident dementia and cognitive decline in older adults: the Health ABC Study. J Gerontol A Biol Sci Med Sci 2017;72(05):703-709

8. Brewster KK, Ciarleglio A, Brown PJet al.. Agerelated hearing loss and its association with depression in later life. Am J Geriatr Psychiatry 2018;26(07):788-796

9. Wu YT, Beiser AS, Breteler MMBet al.. The changing prevalence and incidence of dementia over time - current evidence. Nat Rev Neurol 2017;13(06):327-339

10. Wimo A, Guerchet M, Ali GCet al.. The worldwide costs of dementia 2015 and comparisons with 2010. Alzheimers Dement 2017;13 (01):1-7

11. Hasin DS, Sarvet AL, Meyers JLet al.. Epidemiology of adult DSM-5 major depressive disorder and its specifiers in the United States. JAMA Psychiatry 2018;75(04):336-346

12. Lépine JP, Briley M. The increasing burden of depression. Neuropsychiatr Dis Treat 2011;7 (Suppl 1):3-7

13. Batterham PJ, Christensen $\mathrm{H}$, Mackinnon AJ. Modifiable risk factors predicting major depressive disorder at four year follow-up: a decision tree approach. BMC Psychiatry 2009;9:75

14. Vaisbuch Y, Santa Maria PL. Age-related hearing loss: innovations in hearing augmentation. Otolaryngol Clin North Am 2018;51(04):705-723

15. Yamasoba T, Lin FR, Someya S, Kashio A, Sakamoto T, Kondo K. Current concepts in age-related hearing loss: epidemiology and mechanistic pathways. Hear Res 2013;303:30-38

16. Gates GA, Mills JH. Presbycusis. Lancet 2005; 366(9491):1111-1120

17. Tseng YC, Liu SH, Lou MF, Huang GS. Quality of life in older adults with sensory impairments: a systematic review. Qual Life Res 2018;27(08): 1957-1971

18. Livingston G, Sommerlad A, Orgeta Vet al.. Dementia prevention, intervention, and care. Lancet 2017;390(10113):2673-2734

19. Loughrey DG, Kelly ME, Kelley GA, Brennan S, Lawlor BA. Association of age-related hearing loss with cognitive function, cognitive impairment, and dementia: a systematic review and meta-analysis. JAMA Otolaryngol Head Neck Surg 2018;144(02):115-126
20. Sardone R, Battista P, Panza Fet al.. The agerelated central auditory processing disorder: silent impairment of the cognitive ear. Front Neurosci 2019;13:619

21. Idrizbegovic E, Hederstierna C, Dahlquist M, Rosenhall U. Short-term longitudinal study of central auditory function in Alzheimer's disease and mild cognitive impairment. Dement Geriatr Cogn Disord Extra 2013;3(01):468-471

22. Edwards JD, Lister JJ, Elias MNet al.. Auditory processing of older adults with probable mild cognitive impairment. J Speech Lang Hear Res 2017;60(05):1427-1435

23. Gates GA, Gibbons LE, McCurry SM, Crane PK, Feeney MP, Larson EB. Executive dysfunction and presbycusis in older persons with and without memory loss and dementia. Cogn Behav Neurol 2010;23(04):218-223

24. Quaranta N, Coppola F, Casulli Met al.. The prevalence of peripheral and central hearing impairment and its relation to cognition in older adults. Audiol Neurotol 2014;19(Suppl 1):10-14

25. Gates GA, Beiser A, Rees TS, D'Agostino RB, Wolf PA. Central auditory dysfunction may precede the onset of clinical dementia in people with probable Alzheimer's disease. J Am Geriatr Soc 2002;50(03):482-488

26. Golub JS, Brickman AM, Ciarleglio AJ, Schupf $\mathrm{N}$, Luchsinger JA. Association of subclinical hearing loss with cognitive performance. JAMA Otolaryngol Head Neck Surg 2020;146(01): 57-67

27. Miller G, Miller C, Marrone N, Howe C, Fain M, Jacob A. The impact of cochlear implantation on cognition in older adults: a systematic review of clinical evidence. BMC Geriatr 2015;15:16

28. Völter C, Götze L, Dazert S, Falkenstein M, Thomas JP. Can cochlear implantation improve neurocognition in the aging population? Clin Interv Aging 2018;13:701-712

29. Golub JS. Brain changes associated with agerelated hearing loss. Curr Opin Otolaryngol Head Neck Surg 2017;25(05):347-352

30. Chern A, Golub JS. Age-related hearing loss and dementia. Alzheimer Dis Assoc Disord 2019;33 (03):285-290

31. Rönnberg J, Lunner T, Zekveld Aet al.. The Ease of Language Understanding (ELU) model: theoretical, empirical, and clinical advances. Front Syst Neurosci 2013;7:31

32. Tucker AM, Stern Y. Cognitive reserve in aging. Curr Alzheimer Res 2011;8(04):354-360

33. Taljaard DS, Olaithe M, Brennan-Jones CG, Eikelboom RH, Bucks RS. The relationship between hearing impairment and cognitive function: a meta-analysis in adults. Clin Otolaryngol 2016;41(06):718-729 
34. Bouton K. Living Better with Hearing Loss: A Guide to Health, Happiness, Love, Sex, Work, Friends ... and Hearing Aids. 1st ed.Workman Publishing Company; 2015

35. Strawbridge WJ, Wallhagen MI, Shema SJ, Kaplan GA. Negative consequences of hearing impairment in old age: a longitudinal analysis. Gerontologist 2000;40(03):320-326

36. Mick P, Kawachi I, Lin FR. The association between hearing loss and social isolation in older adults. Otolaryngol Head Neck Surg 2014;150 (03):378-384

37. Poey JL, Burr JA, Roberts JS. Social connectedness, perceived isolation, and dementia: Does the social environment moderate the relationship between genetic risk and cognitive well-being? Gerontologist 2017;57(06):1031-1040

38. Tun PA, McCoy S, Wingfield A. Aging, hearing acuity, and the attentional costs of effortful listening. Psychol Aging 2009;24(03):761-766

39. Wingfield A, Grossman M. Language and the aging brain: patterns of neural compensation revealed by functional brain imaging. J Neurophysiol 2006;96(06):2830-2839

40. Wang HX, Karp A, Winblad B, Fratiglioni L. Late-life engagement in social and leisure activities is associated with a decreased risk of dementia: a longitudinal study from the Kungsholmen project. Am J Epidemiol 2002;155(12):1081-1087

41. Fratiglioni L, Paillard-Borg S, Winblad B. An active and socially integrated lifestyle in late life might protect against dementia. Lancet Neurol 2004;3(06):343-353

42. Dawes P, Emsley R, Cruickshanks KJet al.. Hearing loss and cognition: the role of hearing AIDS, social isolation and depression. PLoS One 2015; 10(03):e0119616

43. Weinstein BE, Sirow LW, Moser S. Relating hearing aid use to social and emotional loneliness in older adults. Am J Audiol 2016;25(01):54-61

44. Peelle JE, Troiani V, Grossman M, Wingfield A. Hearing loss in older adults affects neural systems supporting speech comprehension. J Neurosci 2011;31(35):12638-12643

45. Eckert MA, Cute SL, Vaden KI Jr, Kuchinsky SE, Dubno JR. Auditory cortex signs of age-related hearing loss. J Assoc Res Otolaryngol 2012;13 (05):703-713

46. Lin FR, Ferrucci L, An Yet al.. Association of hearing impairment with brain volume changes in older adults. Neuroimage 2014;90:84-92

47. Geroldi C, Akkawi NM, Galluzzi Set al.. Temporal lobe asymmetry in patients with Alzheimer's disease with delusions. J Neurol Neurosurg Psychiatry 2000;69(02):187-191

48. Armstrong NM, An Y, Doshi Jet al.. Association of midlife hearing impairment with late-life temporal lobe volume loss. JAMA Otolaryngol Head
Neck Surg 2019; (epub ahead of print). Doi: 10.1001/jamaoto.2019.1610

49. Ohlemiller KK. Mechanisms and genes in human strial presbycusis from animal models. Brain Res 2009;1277:70-83

50. Scherer EQ, Yang J, Canis Met al.. Tumor necrosis factor- $\alpha$ enhances microvascular tone and reduces blood flow in the cochlea via enhanced sphingosine-1-phosphate signaling. Stroke 2010; 41(11):2618-2624

51. Yang CH, Schrepfer T, Schacht J. Age-related hearing impairment and the triad of acquired hearing loss. Front Cell Neurosci 2015;9:276

52. Duering $M$, Righart $R$, Csanadi Eet al.. Incident subcortical infarcts induce focal thinning in connected cortical regions. Neurology 2012;79(20): 2025-2028

53. Duering M, Righart R, Wollenweber FA, Zietemann V, Gesierich B, Dichgans M. Acute infarcts cause focal thinning in remote cortex via degeneration of connecting fiber tracts. Neurology 2015; 84(16):1685-1692

54. Ogawa T, Yoshida Y, Okudera T, Noguchi K, Kado H, Uemura K. Secondary thalamic degeneration after cerebral infarction in the middle cerebral artery distribution: evaluation with MR imaging. Radiology 1997;204(01):255-262

55. Hardy J. Testing times for the "amyloid cascade hypothesis”. Neurobiol Aging 2002;23(06): 1073-1074

56. Sinha UK, Hollen KM, Rodriguez R, Miller CA. Auditory system degeneration in Alzheimer's disease. Neurology 1993;43(04):779-785

57. Deal JA, Rawlings A, Sharrett ARet al.. Hearing impairment, cognitive performance, and beta-amyloid deposition in the ARIC-PET Amyloid Imaging Study. Innov Aging 2019;3:551

58. Wilson RS, Yu L, James BD, Bennett DA, Boyle PA. Association of financial and health literacy with cognitive health in old age. Neuropsychol Dev Cogn B Aging Neuropsychol Cogn 2017;24 (02):186-197

59. Jayakody DMP, Friedland PL, Eikelboom RH, Martins RN, Sohrabi HR. A novel study on association between untreated hearing loss and cognitive functions of older adults: Baseline nonverbal cognitive assessment results. Clin Otolaryngol 2018;43(01):182-191

60. Gale SA, Acar D, Daffner KR. Dementia. Am J Med 2018;131(10):1161-1169

61. Lin FR, Ferrucci L, Metter EJ, An Y, Zonderman $\mathrm{AB}$, Resnick SM. Hearing loss and cognition in the Baltimore Longitudinal Study of Aging. Neuropsychology 2011;25(06):763-770

62. Chen SP, Bhattacharya J, Pershing S. Association of vision loss with cognition in older adults. JAMA Ophthalmol 2017;135(09): 963-970 
63. Fischer ME, Cruickshanks KJ, Schubert CRet al.. Age-related sensory impairments and risk of cognitive impairment. J Am Geriatr Soc 2016;64(10): 1981-1987

64. Alves J, Petrosyan A, Magalhães R. Olfactory dysfunction in dementia. World J Clin Cases 2014;2(11):661-667

65. Semenov YR, Bigelow RT, Xue QL, du Lac S, Agrawal Y. Association between vestibular and cognitive function in U.S. adults: data from the National Health and Nutrition Examination Survey. J Gerontol A Biol Sci Med Sci 2016;71(02): 243-250

66. Harun A, Oh ES, Bigelow RT, Studenski S, Agrawal Y. Vestibular impairment in dementia. Otol Neurotol 2016;37(08):1137-1142

67. Huang CQ Dong BR, Lu ZC, Yue JR, Liu QX. Chronic diseases and risk for depression in old age: a meta-analysis of published literature. Ageing Res Rev 2010;9(02):131-141

68. Chen DS, Genther DJ, Betz J, Lin FR. Association between hearing impairment and self-reported difficulty in physical functioning. J Am Geriatr Soc 2014;62(05):850-856

69. Brown PJ, Rutherford BR, Yaffe Ket al.. The depressed frail phenotype: the clinical manifestation of increased biological aging. Am J Geriatr Psychiatry 2016;24(11):1084-1094

70. Gopinath B, Wang JJ, Schneider Jet al.. Depressive symptoms in older adults with hearing impairments: the Blue Mountains Study. J Am Geriatr Soc 2009;57(07):1306-1308

71. Cacciatore F, Napoli C, Abete P, Marciano E, Triassi M, Rengo F. Quality of life determinants and hearing function in an elderly population: Osservatorio Geriatrico Campano Study Group. Gerontology 1999;45(06):323-328

72. Lee AT, Tong MC, Yuen KC, Tang PS, Vanhasselt CA. Hearing impairment and depressive symptoms in an older Chinese population. J Otolaryngol Head Neck Surg 2010;39(05): 498-503

73. Acar B, Yurekli MF, Babademez MA, Karabulut $\mathrm{H}$, Karasen RM. Effects of hearing aids on cognitive functions and depressive signs in elderly people. Arch Gerontol Geriatr 2011;52(03): 250-252

74. Mener DJ, Betz J, Genther DJ, Chen D, Lin FR. Hearing loss and depression in older adults. J Am Geriatr Soc 2013;61(09):1627-1629

75. Poissant SF, Beaudoin F, Huang J, Brodsky J, Lee DJ. Impact of cochlear implantation on speech understanding, depression, and loneliness in the elderly. J Otolaryngol Head Neck Surg 2008;37 (04):488-494

76. Wilson RS, Krueger KR, Arnold SEet al.. Loneliness and risk of Alzheimer disease. Arch Gen Psychiatry 2007;64(02):234-240
77. Chia EM, Wang JJ, Rochtchina E, Cumming RR, Newall P, Mitchell P. Hearing impairment and health-related quality of life: the Blue Mountains Hearing Study. Ear Hear 2007;28 (02):187-195

78. Pronk M, Deeg DJ, Smits Cet al. Prospective effects of hearing status on loneliness and depression in older persons: identification of subgroups. Int J Audiol 2011;50(12):887-896

79. Resnick HE, Fries BE, Verbrugge LM. Windows to their world: the effect of sensory impairments on social engagement and activity time in nursing home residents. J Gerontol B Psychol Sci Soc Sci 1997;52(03):S135-S144

80. Hawton A, Green C, Dickens APet al.. The impact of social isolation on the health status and health-related quality of life of older people. Qual Life Res 2011;20(01):57-67

81. Luo Y, Hawkley LC, Waite LJ, Cacioppo JT. Loneliness, health, and mortality in old age: a national longitudinal study. Soc Sci Med 2012;74 (06):907-914

82. Berkman LF, Glass T, Brissette I, Seeman TE. From social integration to health: Durkheim in the new millennium. Soc Sci Med 2000;51(06): 843-857

83. Cacioppo JT, Hawkley LC. Perceived social isolation and cognition. Trends Cogn Sci 2009;13 (10):447-454

84. Chen DS, Betz J, Yaffe Ket al;Health ABC Study. Association of hearing impairment with declines in physical functioning and the risk of disability in older adults. J Gerontol A Biol Sci Med Sci 2015; 70(05):654-661

85. Gispen FE, Chen DS, Genther DJ, Lin FR. Association between hearing impairment and lower levels of physical activity in older adults. J Am Geriatr Soc 2014;62(08):1427-1433

86. Agmon M, Lavie L, Doumas M. The association between hearing loss, postural control, and mobility in older adults: a systematic review. J Am Acad Audiol 2017;28(06):575-588

87. Grue EV, Ranhoff AH, Noro Aet al.. Vision and hearing impairments and their associations with falling and loss of instrumental activities in daily living in acute hospitalized older persons in five Nordic hospitals. Scand J Caring Sci 2009;23(04): 635-643

88. Jiam NT, Li C, Agrawal Y. Hearing loss and falls: a systematic review and meta-analysis. Laryngoscope 2016;126(11):2587-2596

89. Robins LM, Hill KD, Finch CF, Clemson L, Haines T. The association between physical activity and social isolation in community-dwelling older adults. Aging Ment Health 2018;22(02): 175-182

90. Negrila-Mezei A, Enache R, Sarafoleanu C. Tinnitus in elderly population: clinic correlations 
and impact upon QoL. J Med Life 2011;4(04): 412-416

91. Cianfrone G, Mazzei F, Salviati Met al.. Tinnitus holistic simplified classification (THoSC): a new assessment for subjective tinnitus, with diagnostic and therapeutic implications. Ann Otol Rhinol Laryngol 2015;124(07):550-560

92. Axelsson A, Ringdahl A. Tinnitus-a study of its prevalence and characteristics. Br J Audiol 1989; 23(01):53-62

93. Szibor A, Mäkitie A, Aarnisalo AA. Tinnitus and suicide: an unresolved relation. Audiology Res 2019;9(01):222

94. Ouda L, Profant O, Syka J. Age-related changes in the central auditory system. Cell Tissue Res 2015;361(01):337-358

95. Husain FT, Medina RE, Davis CWet al.. Neuroanatomical changes due to hearing loss and chronic tinnitus: a combined VBM and DTI study. Brain Res 2011;1369:74-88

96. Boyen K, Langers DR, de Kleine E, van Dijk P. Gray matter in the brain: differences associated with tinnitus and hearing loss. Hear Res 2013;295:67-78

97. Helm K, Viol K, Weiger TMet al.. Neuronal connectivity in major depressive disorder: a systematic review. Neuropsychiatr Dis Treat 2018; 14:2715-2737

98. Tokuda T, Yoshimoto J, Shimizu Yet al.. Identification of depression subtypes and relevant brain regions using a data-driven approach. Sci Rep 2018;8(01):14082

99. Peng DH, Jiang KD, Fang YRet al.. Decreased regional homogeneity in major depression as revealed by resting-state functional magnetic resonance imaging. Chin Med J (Engl) 2011;124 (03):369-373

100. Guo WB, Liu F, Xue ZMet al.. Abnormal neural activities in first-episode, treatment-naïve, shortillness-duration, and treatment-response patients with major depressive disorder: a resting-state fMRI study. J Affect Disord 2011;135(13):326-331

101. Rutherford BR, Brewster K, Golub JS, Kim AH, Roose SP. Sensation and psychiatry: linking agerelated hearing loss to late-life depression and cognitive decline. Am J Psychiatry 2018;175 (03):215-224

102. Bruno D, Brown AD, Kapucu A, Marmar CR, Pomara N. Cognitive reserve and emotional stimuli in older individuals: level of education moderates the age-related positivity effect. Exp Aging Res 2014;40(02):208-223

103. Husain FT, Carpenter-Thompson JR, Schmidt SA. The effect of mild-to-moderate hearing loss on auditory and emotion processing networks. Front Syst Neurosci 2014;8:10
104. Hoebel J, Maske UE, Zeeb H, Lampert T. Social inequalities and depressive symptoms in adults: the role of objective and subjective socioeconomic status. PLoS One 2017;12(01):e0169764

105. Golub JS, Brewster KK, Brickman AMet al.. Association of audiometric age-related hearing loss with depressive symptoms among Hispanic individuals. JAMA Otolaryngol Head Neck Surg 2019;145(02):132-139

106. Birrer RB, Vemuri SP. Depression in later life: a diagnostic and therapeutic challenge. Am Fam Physician 2004;69(10):2375-2382

107. Hellewell SC, Welton T, Maller JJet al.. Profound and reproducible patterns of reduced regional gray matter characterize major depressive disorder. Transl Psychiatry 2019;9(01):176

108. Tascone LDS, Payne ME, MacFall Jet al.. Cortical brain volume abnormalities associated with few or multiple neuropsychiatric symptoms in Alzheimer's disease. PLoS One 2017;12(05):e0177169

109. Fang P, Zeng LL, Shen Het al.. Increased cortical-limbic anatomical network connectivity in major depression revealed by diffusion tensor imaging. PLoS One 2012;7(09):e45972

110. Korgaonkar MS, Cooper NJ, Williams LM, Grieve SM. Mapping inter-regional connectivity of the entire cortex to characterize major depressive disorder: a whole-brain diffusion tensor imaging tractography study. Neuroreport 2012;23 (09):566-571

111. Campbell J, Sharma A. Compensatory changes in cortical resource allocation in adults with hearing loss. Front Syst Neurosci 2013;7:71

112. Bennett S, Thomas AJ. Depression and dementia: cause, consequence or coincidence? Maturitas 2014;79(02):184-190

113. Mosnier I, Vanier A, Bonnard Det al.. Long-term cognitive prognosis of profoundly deaf older adults after hearing rehabilitation using cochlear implants. J Am Geriatr Soc 2018;66(08): 1553-1561

114. Mosnier I, Bebear JP, Marx Met al.. Improvement of cognitive function after cochlear implantation in elderly patients. JAMA Otolaryngol Head Neck Surg 2015;141(05):442-450

115. Brent RJ. A cost-benefit analysis of hearing aids, including the benefits of reducing the symptoms of dementia. Appl Econ 2019;51(28):3091-3103

116. Willink A, Reed NS, Lin FR. Cost-benefit analysis of hearing care services: What is it worth to Medicare? J Am Geriatr Soc 2019;67(04): 784-789

117. Yong M, Willink A, McMahon Cet al.. Access to adults' hearing aids: policies and technologies used in eight countries. Bull World Health Organ 2019;97(10):699-710 